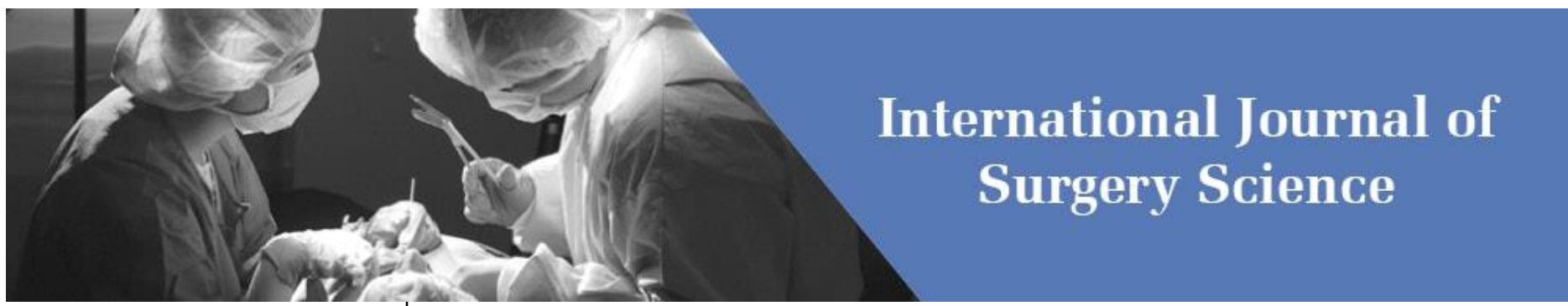

E-ISSN: 2616-3470

P-ISSN: 2616-3462

(C) Surgery Science

www.surgeryscience.com

2020; 4(1): 314-315

Received: 19-11-2019

Accepted: 23-12-2019

Dr. Anil Kumar MS

Professor, Department of General Surgery, JSS Hospital Mysore,

Karnataka, India

Dr. Surendra Mannan

Professor, Department of General

Surgery, JSS Hospital Mysore,

Karnataka, India

\section{A rare case of rectal neuroendocrine tumor presenting as a polyp}

\section{Dr. Anil Kumar and Dr. Surendra Mannan}

DOI: https://doi.org/10.33545/surgery.2020.v4.i1f.357

\section{Abstract}

This is a case reporting regarding a patient presenting with gluteal abscess underwent Incision and drainage and intraoperatively solitary rectal polyp was found on proctoscopy examination, excision(polypectomy) was done and sent for histopathological examination, which was found to be a well differentiated tumour with tumour cells expressing cytokeratin and synaptophysin and were immunonegative for chromogranin A. Margins of the specimen showed free of tumor cells, and there was no distant metastasis. A follow up colonoscopy was done after 6 months which showed normal findings.

Keywords: NET, neuroendocrine tumor, gastrointestinal system, vasoactive peptide harmones, ki-67 index

\section{Introduction}

Neuroendocrine tumors originate from neuroendocrine cells of endodermal origin, spreading along the gastrointestinal tract. Incidence of rectal NET is $0.93 / 100000$ and has been increasing in the last decade partly due to improved screening for colorectal diseases. According to the WHO classification all NET's are to be considered as if they had malignant potential. They are most frequently observed in gastrointestinal system (GIS) [67\%]. In GIS, they are most frequently observed at ileum [34\%] and secondly at rectum [27.4\%].

Neuroendocrine tumors excrete various peptide hormones such as chromogranin A, pancreatic polypeptide, calcitonin and substance $\mathrm{P}$, which maybe biologically active and biological amines like neuron specific enolase, serotonin, 5-hydroxy tryptamine are under the control of autonomic nervous system. Tumors with size less than one centimeter have a low risk for metastasis. This risk increases to $60-80 \%$ at tumors with size greater than $2 \mathrm{~cm}$.

Table 1: Tumor grade according to mitotic count and proliferation

\begin{tabular}{|c|c|c|}
\hline WHO grade & Mitoticount (10HF1PF) & Ki-67 index \\
\hline G1 & $<2$ & $<2 \%$ \\
\hline G2 & $2-20$ & $3-20 \%$ \\
\hline
\end{tabular}

Table 1: WHO Tumor grade is based on mitotic count and on proliferation (Ki-67 index) and the three levels defined are described here.

\section{Case report}

\section{History}

A 64 year old male patient presented with swelling in the perianal region for 4 days associated with throbbing type of pain. He also gave history of pain during defecation. He did not give any history of altered bowel habits or bleeding per rectum. No h/o other co morbidities.

\section{Examination}

General condition of the patient defined well and his vital signs were within the normal range (Arterial tension: $110 / 80 \mathrm{mmHg}$, pulse: $82 / \mathrm{min}$ ). On clinical examination, a solitary swelling $4 \mathrm{x}$ $4 \mathrm{~cm}$ was present over the perianal region. Skin over the swelling was erythematous. Tenderness and local rise of temperature was present. The swelling was soft, fluctuant and induration was present in the area surrounding the swelling.
Professor, Department of General Surgery, JSS Hospital Mysore,

Karnataka, India 
A working diagnosis of Ischiorectal abscess was made and patient was taken up for emergency incision and drainage. Intraoperatively, per rectal examination under anesthesia, suspicious nodule was present and proctoscopy was done which revealed a solitary polyp of $1-2 \mathrm{~cm}$ at 3 o'clock position present around $7 \mathrm{~cm}$ from the anal verge.

\section{Proctoscope}

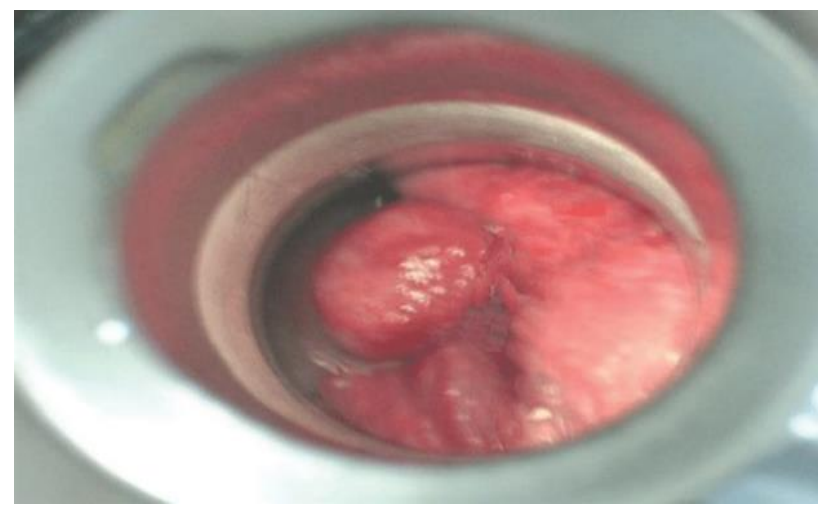

Fig 1: Proctoscope examination under anesthesia shows a polyp.

\section{Treatment}

Incision and drainage along with polypectomy was done and the specimen was sent for histopathological examination.

\section{HPE report}

Macro: Single polypoidal grey white tissue mass of $1.5 \times 1.5$ cms.

\section{Micro}

- Polypoidal lesion lined by rectal mucosa.

- The submucosa and muscular layer show neuroendocrine cells arranged predominantly in glandular pattern.

- The resected margins were free of the tumor cells.

- Mitotic rate <2 per hpf, PAS NEGATIVE.

- IMPRESSION-Neuroendocrine tumor, WHO Grade 1.

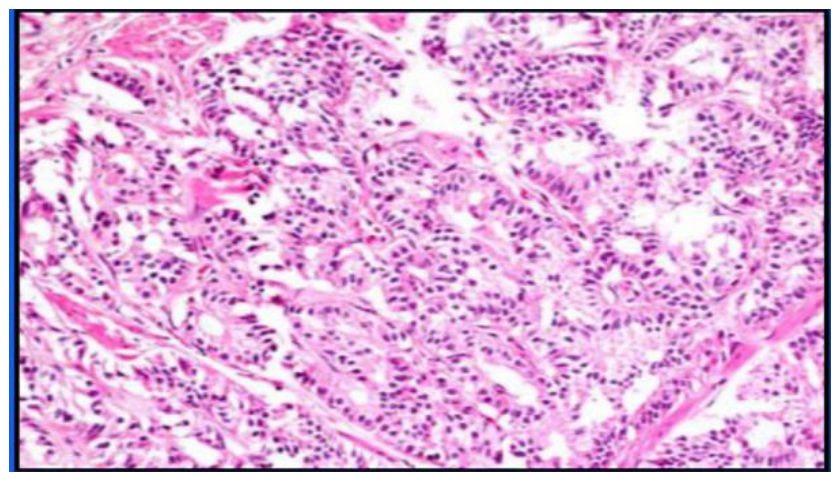

Fig 2: HPE of Polyp

\section{Immunohistochemistry}

The specimen was found to be a well differentiated tumor, with tumor cells expressing cytokeratin and synaptophysin and were immunonegative for chromogranin A.

A follow up colonoscopy was done after 6 months which showed normal findings.

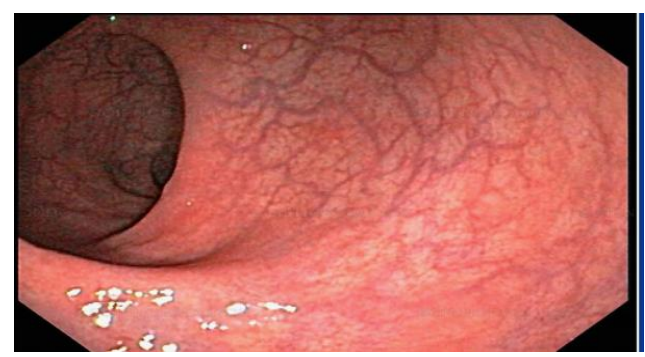

Fig 3: Colonoscopy (Follow UP)

\section{Discussion}

The increased rate of detection of rectal NETs can be contributed to the increased awareness among both clinician and pathologist. These tumors are generally asymptomatic and present as polyps on colonoscopies. Slight male predominance and mean age at diagnosis is 57 years. Rectal NETs have a higher incidence and prevalence in both African American and Asian populations as compared to Caucasian. EUS can play a major role in pre-operative planning and is recommended to assess tumor size, depth of invasion, and the presence of lymph node involvement.

The ones with size less than one centimeter generally have a benign clinical course, metastasis may also be observed rarely. Treatment of NETs of size between 1 and $1.9 \mathrm{~cm}$ is controversial. Metastasis rate is $4-30 \%$ in these tumors and it is difficult to predict their behavior. Tumors with size greater than two centimeters may have an aggressive course and poor prognosis. Metastasis can be detected in 70-80\% during followup or at the time of diagnosis. Somatostatin receptor analogs are used in immunotherapy at patients with distant metastasis. IFN (interferon) is considered to be a cost-effective treatment. PRRT (peptide receptor radio-nucleotide therapy) appears to be a key strategy in terms of treatment and imaging.

In this case, the polyp was an intraoperative incidental finding and polypectomy was done at the same sitting. As the HPE report showed that the margins were free of tumor even though the tumor was extending up to muscular layer and there was no signs of distant metastases, no further resection was required.

\section{Conclusion}

- WHO staging should be performed to determine an appropriate treatment approach according to the 2010 guidelines.

- A plethora of minimally invasive endoscopic interventions for resection of rectal NETs have emerged.

- Prospective studies are needed to adequately compare the endoscopic intervention options available and their effects on patient outcomes.

\section{References}

1. Rakici H, Akdogan RA, Yurdakul C, Canturk N. A case of rectal neuroendocrine tumor presenting as polyp. International Journal of Surgery case reports. 2015; 8:59-61.

2. Chablaney S, Zator ZA, Kumta NA. Diagnosis and management of rectal neuroendocrine tumors. Clinical endoscopy. 2017; 50(6):530.

3. Rodrigues Â, Castro-Poças F, Pedroto I. Neuroendocrine Rectal Tumors: Main Features and Management. GE Portuguese journal of gastroenterology. 2015; 22(5):213. 